\title{
COMPLICAÇÕES DECORRENTES DA UTILIZAÇÃO DA ACEPROMAZINA ASSOCIADA À XILAZINA NA PREPARAÇÃO CIRÚRGICA DE RUFIÕES BOVINOS
}

\author{
RESULTS COMPLICATIONS OF ACEPROMAZINE AND XYLAZINE \\ IN SURGICAL PREPARATION OF TEASER BULLS
}

\section{Luiz Antônio Franco da Silva ${ }^{1}$ Sibele Martins Chaves $^{2}$ Maria Clorinda Soares Fioravanti $^{3}$ Duvaldo Eurides $^{4}$ Rogério Elias Rabelo ${ }^{5}$}

\section{RESUMO}

$O$ presente estudo teve a finalidade de avaliar, em propriedades rurais, os períodos trans e pós-operatório de rufiões bovinos preparados pelas técnicas cirúrgicas de desvio do pênis e de formação de um novo óstio prepucial, utilizando como tranqüilizante a xilazina ou a associação entre a xilazina e a acepromazina, acrescidos de anestesia local. Foram utilizados 156 bovinos machos, com idade entre 12 e 18 meses, sendo que 108 foram submetidos ao desvio do pênis e 48 operados pela técnica de formação de um novo óstio. Os bovinos foram alocados aleatoriamente em dois grupos compostos por 78 animais cada (um grupo recebendo somente xilazina; o outro, a associação xilazinalacepromazina, ambos recebendo anestesia local com lidocaína 2\%). Durante 30 dias do pós-operatório, os bovinos foram avaliados semanalmente. Foram observadas complicações no período pós-operatório em 72 animais (92,31\%) quando se utilizou a associação medicamentosa, e em 29 bovinos $(37,18 \%)$ tranqüilizados com a xilazina considerando-se as duas técnicascirúrgicas utilizadas. $O$ teste de $\chi^{2}$ mostrou que a associação medicamentosa resultou em aumento das complicações pós-operatórias.

Palavras-chave: rufiões bovinos, xilazina, acepromazina, pósoperatório.

\section{SUMMARY}

The objective of this study was to evaluate, in farms, the trans and postoperative periods of teaser bulls prepared by the surgical techniques of lateral deviation of prepuce and penis (lateral deviation) and lateral deviation with a fixation of the preputial mucosa to a new orifice (new orifice), using as tranquilizer xylazine or xylazine/acepromazine association, added of local anesthesia. 156 bulls, at 12 to 18 months of age were used; 108 bulls were submitted to lateral deviation of prepuce and penis and 48 to new orifice. The bulls were casually allocated in two groups composed by 78 animals (a group receiving only xylazine, another group receiving xylazine/acepromazine association, both receiving local anesthesia with lidocayne $2 \%$ ). The bulls were weekly appraised, by thirty days. Xylazine/acepromazine association resulted in postoperative complications in 72 animals $(92,31 \%)$ and xylazine resulted in postoperative complications in 29 animals $(37,18 \%)$. The $\chi^{2}$ test showed that Xylazinelacepromazine association resulted in increase of postoperative complications.

Key words: teaser bulls, xylazine, acepromazine, postoperative.

\section{INTRODUÇÃO}

Os avanços tecnológicos observados na pecuária durante as últimas décadas, indicam a necessidade de desenvolver procedimentos operatórios eficientes e econômicos.

CLAXTON (1989) relacionou diferentes métodos de preparação cirúrgica de rufiões; SMITH (1967) e OHEME (1968) propuseram, inicialmente, para se obter rufiões bovinos, apenas a esterilização do touro, porém permitindo a realização da cópula. Outros métodos que impediam a cópula, como a

\footnotetext{
${ }^{1}$ Médico Veterinário, professor da Clínica Cirúrgica Animal da Escola de Veterinária (EV), Universidade Federal de Goiás (UFG). Endereço: Rua 18-A, 591, ap. 502, Setor Aeroporto, 74.070-060, Goiânia, Goiás. Autor para correspondência.

${ }^{2}$ Médico Veterinário, aluno do curso de mestrado em Sanidade Animal da EV, UFG.

${ }^{3}$ Médico Veterinário, professor da Clínica da EV, UFG.

${ }^{4}$ Médico Veterinário, professor da Cirurgia Animal da Universidade Federal de Uberlândia.

${ }^{5}$ Médico Veterinário, aluno do curso de mestrado em Sanidade Animal da EV, UFG.
} 
aderência do pênis (BELLING, 1961), a retroflexão (JILLELLA et al., 1978), o desvio lateral do pênis (ROMMEL, 1961, SCHULTZ et al., 1999), o desvio ventral do pênis (OEHME, 1988), a formação de fundo de saco prepucial (SMITH, 1963), ou até mesmo a falectomia (STRAUB \& KENDRICK, 1965) também foram propostos. CARNEIRO (1973) promoveu o desvio lateral do pênis com formação de um novo óstio prepucial e EURIDES et al. (1992) desenvolveram técnica cirúrgica com remoção do ligamento apical do pênis. A técnica de Monroy (fixação da flexura sigmóide do pênis) foi descrita por EURIDES (1981) e MOLINARI et al. (1998).

OEHME (1988) recomenda a adição da vasectomia ou da remoção da cauda do epidídimo às técnicas cirúrgicas que impedem a cópula, para assegurar a ausência de prenhez caso haja cópula acidental. TEGEGNE et $\boldsymbol{a l}$. (1993) desenvolveram um estudo utilizando animais vasectomisados na detecção do cio de vacas e LOPEZ et al. (1999) avaliaram a epididimectomia como método de preparação de rufiões bubalinos.

O uso somente de anestesia local no preparo de rufiões bovinos foi citada por SMITH (1967). STRAUB \& KENDRICK (1965) optaram pela anestesia epidural lombar e CARNEIRO (1973) afirma ter conseguido resultados satisfatórios apenas com o uso de cloridrato de xilazina na dose de $0,3 \mathrm{mg} / \mathrm{kg}$.

CRONIN et al. (1983) apontaram a necessidade de discutir as associações anestésicas nas diferentes espécies, para encontrar aquela que seja eficiente e menos onerosa. Os autores estudaram os efeitos da associação acepromazina/xilazina em cães nas doses respectivas de $2,2 \mathrm{mg} / \mathrm{kg}$ e $0,5 \mathrm{mg} / \mathrm{kg}$ e demonstraram que essa combinação produz um estado de relaxamento, imobilização profunda, sedação e moderada analgesia sendo, portanto, muito útil como préanestésico e na execução de pequenas cirurgias.

São várias as complicações pósoperatórias que podem ser observadas quando são empregadas diversas técnicas de preparo de rufiões bovinos. BELLING (1961) e SMITH (1963) relataram o represamento de urina na cavidade prepucial e a dificuldade de exposição do pênis no momento da micção, quando se empregam de técnicas como a aderência do pênis à parede abdominal e a formação de fundo de saco prepucial. De acordo com CARNEIRO (1973), o emprego da técnica de formação de novo óstio prepucial favoreceu uma discreta hemorragia observada nos animais jovens e mais acentuada nos mais velhos, além de ligeiro edema nos primeiros dias do período pós-operatório.
A acepromazina em bovinos machos é utilizada como meio auxiliar na exposição do pênis (LEITE RIBEIRO et al., 1984). Já a xilazina vem sendo utilizada como um dos meios eficazes de se sedar bovinos a serem preparados cirurgicamente para a rufiação, porém resulta em salivação, depressão e, para SILVA et al. (2000), reduz os movimentos ruminais.

O presente estudo teve a finalidade de avaliar, em propriedades rurais, os períodos trans e pós-operatório de rufiões bovinos preparados pelas técnicas cirúrgicas de desvio do pênis e de formação de um novo óstio prepucial, em que se utilizou como tranqüilizante a xilazina ou a associação entre a xilazina e a acepromazina, acrescidos de anestesia local.

\section{MATERIAL E MÉTODOS}

Foram utilizados 156 bovinos machos, com idade entre 12 e 18 meses, com pesos entre 180 e 300kg da raça Girolanda, em bom estado geral, operados em diferentes propriedades rurais do Estado de Goiás, no período compreendido entre 1997 e 2000.

Realizaram-se as intervenções em fazendas, sendo que 108 bovinos foram submetidos à cirurgia para desvio do óstio prepucial utilizando a técnica de ROMMEL (1961), modificada por SILVA et al. (2000) e os outros 48 animais foram operados pela técnica de formação de um novo óstio prepucial, segundo metodologia proposta por CARNEIRO (1973). As intervenções cirúrgicas foram realizadas por um único profissional. A modificação da técnica de ROMMEL (1961) constou de confecção de um túnel para realizar o desvio, em vez de incisões em "V" propostas na técnica original. Os bovinos foram alocados aleatoriamente em dois grupos compostos por 78 animais cada, sendo que um grupo recebeu somente xilazina e o outro recebeu a associação xilazina/acepromazina. Os animais de ambos os grupos foram, também, submetidos à anestesia local. Do grupo que recebeu somente a xilazina (Grupo 1) como tranqüilizante, 54 animais foram submetidos à técnica de ROMMEL (1961) modificada, e 24 foram submetidos à técnica de CARNEIRO (1973). Essa mesma distribuição foi mantida para o grupo dos bovinos que receberam a associação xilazina/acepromazina (Grupo 2).

O pré-operatório constou de jejum completo por um período de 18 horas. Os animais do grupo 1 receberam $0,2 \mathrm{mg} / \mathrm{kg}$ de xilazina $^{\mathrm{a}}$, via intramuscular, e os do grupo 2 receberam a associação xilazina/acepromazina ${ }^{\mathrm{b}}$, na dose de $0,1 \mathrm{e}$ $0,05 \mathrm{mg} / \mathrm{kg}$, respectivamente. A contenção foi 
realizada em decúbito lateral direito e o campo operatório foi tricotomizado e higienizado com solução a 3:1000 de iodofor ${ }^{\mathrm{c}}$. A anestesia local foi realizada por infiltração subcutânea de lidocaína ${ }^{\mathrm{d}}$ a $2 \%$ na região das incisões. Na técnica de desvio do óstio prepucial, a substância anestésica foi infiltrada circundando o óstio prepucial original e na região do abdômen, onde se removeu a pele para transferência do óstio. Na técnica de formação de novo óstio prepucial, a anestesia local ficou restrita à região do prepúcio, onde o novo óstio foi confeccionado.

No período pós-operatório, os animais receberam cinco aplicações de penicilina $G$ benzatina (3.000.000 de UI), penicilina G potássica (1.500.000 de UI), penicilina G procaína ${ }^{\mathrm{e}}$ (1.500.000 de UI), via intramuscular, de dois em dois dias, na dose de 20.000 UI/kg PV e escinato de $\operatorname{sódio}^{f}$, via intravenosa, na dose de $5,1 \mathrm{mg} / 70 \mathrm{~kg}$, durante cinco dias (MARQUES et al., 1988). A ferida cirúrgica foi tratada com óxido de zinco a $18 \%$, óleo de pinho a $5 \%$, caulin a $24 \%$ e xilol $^{\mathrm{g}}$ a $5,5 \%$, nos rufiões obtidos por meio do desvio lateral; nos demais, a ferida foi lavada utilizando-se uma solução a 3:1000 de iodofor, para em seguida aplicar-se uma pomada a base de nitrofurazona ${ }^{\mathrm{h}}$, até completar a cicatrização.

Durante 30 dias subseqüentes à cirurgia, os bovinos foram avaliados semanalmente. A retirada dos pontos foi praticada entre o $10^{\circ}$ e o $14^{\circ}$ dias do período pós operatório, de forma alternada, considerando sempre a cicatrização clínica. Recomendou-se a separação dos animais operados das vacas e a utilização dos mesmos na rufiação somente após o período de 60 dias.

As análises estatísticas foram obtidas através do teste de $\chi^{2}$, utilizando-se a tabela de contingência. Foram feitas comparações entre as duas técnicas cirúrgicas, utilizando-se primeiramente a xilazina e posteriormente a associação xilazina + acepromazina na tranqüilização; e a comparação entre os dois métodos de tranqüilização, independente da técnica cirúrgica empregada (SAMPAIO, 1998).

\section{RESULTADOS E DISCUSSÃO}

O jejum alimentar e hídrico de 18 horas foi importante, pois, além de prevenir o timpanismo e a regurgitação, reduziu a possibilidade de micção durante a cirurgia, corroborando com as observações de CARNEIRO (1973).

Quando se utilizou a associação xilazina/ acepromazina ocorreram $17(23,61 \%)$ casos de prolapso peniano ou de mucosa, contra apenas 2 $(6,9 \%)$ do total de complicações para os animais que receberam somente xilazina (Tabela 1). Esta observação confirma os achados de LEITE RIBEIRO et al., (1984), os quais obtiveram a exposição peniana em bovinos utilizando acepromazina. A acepromazina também foi utilizada por SILVA (1992), na dose de 10mg/100kg PV com bons resultados, na preparação de rufiões eqüinos através da técnica de circuncisão e encurtamento do pênis, para facilitar a exposição peniana durante o pós-operatório.

A associação medicamentosa utilizada resultou em algum tipo de complicação no período pós-operatório em 72 animais $(92,31 \%)$, contra apenas 29 bovinos $(37,18 \%)$ tranqüilizados somente com a xilazina (Tabela 1). Por outro lado, esses achados demonstram que o método de tranqüilização ou de anestesia utilizado ainda é hoje uma preocupação tão importante quanto a técnica cirúrgica de eleição na preparação de rufiões bovinos. EURIDES (1981) lembra que, nos métodos de preparação de detectores de cio, descritos na literatura, não se observa uniformidade quanto à escolha da anestesia empregada. $\mathrm{O}$ autor utilizou cloridrato de xilazina, $0,2 \mathrm{mg} / \mathrm{kg}$, via intramuscular, associado à anestesia local com lidocaína a $2 \%$, argumentando que a ação da xilazina facilitou a contenção, enquanto o anestésico local impediu as manifestações dolorosas, facilitando a cirurgia. Já CRONIN et al. (1983), estudando os efeitos da associação acepromazina/xilazina em cães, asseguram que a maior vantagem da associação, devese ao fato de a mesma poder ser usada sem riscos, pois é convenientemente antagonizada pelo 4-aminopiridina e/ou yohimbine, sendo as doses utilizadas no experimento de $0,5 \mathrm{mg} / \mathrm{kg}$ e $0,25 \mathrm{mg} / \mathrm{kg}$, respectivamente.

Dos oito casos de prolapsos de mucosa e do pênis (um caso no grupo 1 e sete no grupo 2) em que, devido à necrose, foi necessária a amputação, sete $(9,72 \%$ do total de complicações do grupo 2$)$ (Tabela 1) podem ser atribuídos, em parte, ao efeito da acepromazina, responsável pela exposição prolongada do pênis e/ou mucosa, que facilita o ressecamento, o comprometimento da circulação local e, conseqüentemente, o aparecimento de ferimentos, edema e isquemia. LEITE RIBEIRO $\boldsymbol{e} \boldsymbol{t}$ al. (1984), utilizando apenas a acepromazina a $1 \%$ como meio auxiliar na exposição de pênis bovino, na dose de $0,035 \mathrm{mg} / \mathrm{kg}$ de peso corporal, sem submeter os animais a qualquer intervenção cirúrgica, obtiveram resultados satisfatórios e não observaram efeitos colaterais. No presente estudo, além de associar a acepromazina com a xilazina, a dose utilizada do primeiro fármaco foi maior que a citada pelos autores, uma vez que se pretendia avaliar o efeito tranquiilizante da associação e não a exposição do pênis decorrente da ação da acepromazina. 
Tabela 1 - Complicações pós-operatórias em rufiões bovinos preparados pelas técnicas cirúrgicas de desvio lateral do pênis e novo-óstio prepucial, nos quais foi rutilizado como tranqüilizante a xilazina ou a combinação xilazina/acepromazina, durante o período de 1997 a 2000, em diferentes propriedades rurais do Estado de Goiás.

\begin{tabular}{|c|c|c|c|c|c|c|}
\hline \multirow{3}{*}{$\begin{array}{l}\text { Complicações } \\
\text { pós-operatórias }\end{array}$} & \multicolumn{3}{|c|}{ Tranqüilização com xilazina } & \multicolumn{3}{|c|}{ Tranqüilização com xilazina/acepromazina } \\
\hline & \multicolumn{3}{|c|}{ Técnicas cirúrgicas } & \multicolumn{3}{|c|}{ Técnicas cirúrgicas } \\
\hline & $\begin{array}{c}\text { Desvio lateral do } \\
\text { pênis }\end{array}$ & Novo óstio prepucial & Total & Desvio lateral do pênis & Novo óstio prepucial & Total \\
\hline $\begin{array}{l}\text { Deiscência parcial de } \\
\text { ferida }\end{array}$ & 02 & 03 & 05 & 06 & 03 & 09 \\
\hline Edema de diferentes graus & 06 & 08 & 14 & 17 & 09 & 26 \\
\hline $\begin{array}{l}\text { Estenose do óstio com } \\
\text { necrose e sacrifício }\end{array}$ & 00 & 01 & 01 & 02 & 00 & 02 \\
\hline $\begin{array}{lll}\text { Estenose do óstio } & \text { com } \\
\text { recuperação } & & \text { após } \\
\text { reintervenção } & & \end{array}$ & 01 & 02 & 03 & 01 & 02 & 03 \\
\hline $\begin{array}{l}\text { Prolapso da mucosa } \\
\text { prepucial com recuperação }\end{array}$ & 01 & 00 & 01 & 10 & 00 & 10 \\
\hline $\begin{array}{l}\text { Prolapso da } \text { mucosa } \\
\text { prepucial com necrose e } \\
\text { amputação }\end{array}$ & 00 & 00 & 00 & 02 & 00 & 02 \\
\hline $\begin{array}{l}\text { Prolapso peniano com } \\
\text { necrose e amputação }\end{array}$ & 00 & 01 & 01 & 03 & 02 & 05 \\
\hline Deiscência total da ferida & 01 & 00 & 01 & 05 & 02 & 07 \\
\hline $\begin{array}{l}\text { Abcessos subcutâneos na } \\
\text { região do túnel }\end{array}$ & 02 & 01 & 03 & 06 & 02 & 08 \\
\hline Total & 13 & 16 & 29 & 52 & 20 & 72 \\
\hline
\end{tabular}

Do total de complicações de todos os bovinos operados, a estenose do óstio com necrose e posterior sacrifício ocorreu em três animais $(2,97 \%)$; a estenose do óstio com recuperação após intervenção em seis $(5,94 \%)$ e os abcessos subcutâneos na região do túnel, em 11 (10,89\%) bovinos (Tabela 1).

A ocorrência de prolapso do folheto prepucial interno com posterior recuperação em 11 bovinos representou $10,89 \%$ das complicações de todos os animais operados (Tabela 1). Essa recuperação deveu-se, provavelmente, ao uso do escinato de sódio nesses animais, por um período de dez dias, associado à aplicação diária de uma pomada à base de nitrofurazona que, além da ação antimicrobiana, teve a função de auxiliar na remoção das escamas que se formaram sobre as feridas e de amenizar o ressecamento da mucosa. JENNINGS (1984), ROMMEL (1961), JOCHELE et al. (1973) apontaram o edema como a principal complicação no período pós-operatório de rufiões preparados pelo desvio lateral do pênis, mas não relataram a conduta adotada com vistas à recuperação.

As propriedades rurais utilizadas nesse estudo refletem as condições de manejo e higiene da grande maioria das fazendas da região e o período pós-operatório, geralmente, fica sob a responsabilidade da mão de obra auxiliar ou de Médicos Veterinários que assistem as propriedades. Apesar de o maior número de complicações ter ocorrido quando se utilizou a associação xilazina/acepromazina, independente da intervenção cirúrgica utilizada, é provável que a presença de edemas graves em 40 animais $(39,60 \%)$ e deiscência de feridas em 22 animais $(21,78 \%)$, (Tabela 1) possa ter sido agravada por fatores como higiene deficiente das instalações, falhas de conduta no período pós-operatório e a manutenção dos rufiões com fêmeas antes da recuperação clínica se completar.

Observando as complicações pósoperatórias notou-se que os prolapsos de mucosa estiveram presentes apenas quando se utilizou a técnica de desvio lateral, fato não observado quando se aplicou a técnica de formação de um novo óstio prepucial. Este achado reafirma a importância da redução da lâmina interna do prepúcio, defendida por CARNEIRO (1973).

A associação de tranqüilizantes utilizada vem confirmar as afirmações de CRONIN et al. (1983), os quais trabalhando com diferentes espécies animais obtiveram um estado de sedação, 
relaxamento e imobilização mais profundos quando associaram a xilazina com a acepromazina. No presente estudo, preferiu-se não utilizar apenas a xilazina como tranqüilizante conforme recomenda CARNEIRO (1973) por não parecer suficiente para impedir manifestações dolorosas. Preferiu-se seguir as recomendações de EURIDES (1981), associandose um bloqueio anestésico local nas duas formas de tranqüilização empregadas. Todavia, tomou-se o cuidado com a dose do anestésico local empregado temendo-se a infiltração do excesso nos interstícios teciduais, fato que poderia contribuir para o aparecimento de edemas, conforme menciona SILVA (1992).

Quando se utilizou a associação acepromazina/xilazina, além do aspecto segurança durante o ato cirúrgico, verificou-se que o efeito deambulatório foi reduzido com a recuperação mais rápida dos bovinos após a intervenção cirúrgica, além de não serem observadas maiores complicações no quadro cardio-respiratório dos animais, apontadas por CRONIN et al. (1983) e NILSFORS et al. (1988). Como a associação medicamentosa promove recuperação mais rápida dos animais, os fatores negativos citados por LOPEZ et al. (1999) são amenizados.

$$
\text { Utilizando-se a associação }
$$

xilazina/acepromazina, tornou-se dispensável o uso de dose de manutenção durante a cirurgia. Já a tranqüilização realizada usando-se apenas a xilazina requereu doses complementares em 18,5\% (10) dos animais submetidos à técnica de ROMMEL e $12,5 \%$ (3) dos animais submetidos à técnica de CARNEIRO (1973). Esse autor e EURIDES (1981) utilizaram a xilazina na preparação cirúrgica de touros excitadores, mas não apontaram a necessidade de realizar doses adicionais de manutenção.

Comparando-se as técnicas de ROMMEL (1961) modificada e CARNEIRO (1973), quando utilizada a xilazina como tranqüilizante, foi obtido um $\chi^{2}$ calculado $=12,92$, que é maior que o $\chi^{2} \cdot 05(1)=$ 3,84 , indicando a existência de diferença significativa ( $5 \%$ de probabilidade) entre as técnicas com relação à ocorrência de complicações pósoperatórias. A técnica de ROMMEL (1961) modificada, quando realizada com tranqüilizante xilazina, proporcionou uma melhor cirurgia, com $75,93 \%$ (41 animais) de recuperação sem complicações pós-cirúrgicas.

Utilizando-se a associação xilazina + acepromazina na tranqüilização dos animais e comparando a técnica de ROMMEL (1961) modificada e a de CARNEIRO (1973) encontrou-se um $\chi^{2}$ calculado $=3,91$, que é maior que o $\chi^{2} \cdot 05(1)=$ 3,84 , o que permite afirmar que existe diferença significativa ( $5 \%$ de probabilidade) entre as técnicas com relação à ocorrência de complicações pósoperatórias. A técnica de CARNEIRO (1973), quando realizada com tranqüilizante xilazina + acepromazina, proporcionou uma melhor cirurgia, com 16,67\% (4 animais) de recuperação sem complicações pós-cirúrgicas, diferindo significativamente da técnica de ROMMEL (1961). Na comparação entre os métodos de tranqüilização xilazina , e xilazina + acepromazina , independente da técnica cirúrgica, foi obtido um $\chi^{2}$ calculado $=51,92$, que é maior que o $\chi^{2}{ } \cdot 05(1)=3,84$. Isso permite afirmar que existe diferença significativa $(5 \%$ de probabilidade) entre os métodos de tranqüilização com relação à ocorrência de complicações pósoperatórias. A xilazina proporcionou menor número de complicações, com 62,82\% (49 animais) de recuperação sem qualquer problema. $\mathrm{Na}$ associação xilazina + acepromazina, pôde-se observar 92,31\% (72 animais) com complicações pós-cirúrgicas.

\section{CONCLUSÕES}

Utilizando-se a associação da xilazina com a acepromazina, é possível preparar cirurgicamente rufiões bovinos, em propriedades rurais de forma segura para o animal e para o cirurgião.

A associação da xilazina com a acepromazina aumenta as complicações no pósoperatório de rufiões bovinos preparados pelas intervenções cirúrgicas de desvio e formação de novo óstio prepucial.

\section{FONTES DE AQUISIÇÃO}

${ }^{a}$ ROMPUM - Bayer do Brasil S/A - São Paulo - S.P.

${ }^{\mathrm{b}}$ ACEPRAN $1 \%$ - Univet S/A Ind. Vet.- São Paulo -S.P.

${ }^{\mathrm{c}}$ BIOCID - Lab. Pfizer Ltda - Div. Agrop. - Guarulhos - S.P.

${ }^{\mathrm{d}}$ LIDOCAÍNA $2 \%$ - Instituto Vital Brasil S/A- Niterói - R.J.

e PENCIVET SUPER FORTE PLUS - Quimio Divisão Veterinária - Rio de Janeiro - R.J.

${ }^{\mathrm{f}}$ REPARIL-BYK - Química e Farmacêutica S/A - Rio de Janeiro - R.J.

${ }^{\mathrm{g}}$ UNGÜENTO PEARSON - Pearson Ind. e Com. Ltda - Rio de Janeiro - R.J.

${ }^{\text {h }}$ FURACIM POMADA - Indústria Quim. e Farmacêutica Schering-Plough S/A - Rio de Janeiro - R.J.

\section{REFERÊNCIAS BIBLIOGRÁFICAS}

BELLING , T.H. Preparation of a "Teaser" bull for use in a beef cattle artificial insemination program. J Am Vet Med Assoc, v.138, n.12, p.670-672, 1961.

CARNEIRO, M.I. Preparação de rufiões bovinos. Técnica cirúrgica para formação de neo-óstio prepucial. Belo Horizonte, 1973. 20p. Dissertação (Mestrado em Cirurgia) Escola de Veterinária da UFMG, 1973. 
CLAXTON, M.S. Methods of surgical preparation of teaser bulls. Comp Cont Educ Pract Vet - Food Animal, v.11, n.8, p.974-981, 1989

CRONIN, M.F., BOOTH, N. H., HATCH, R.C., et al Acepromazine-xylazine combination in dogs: antagonism with 4 - aminopyredine and yohimbine. Am J Vet Res, v. 44, n.11, p.2037-2042, 1983.

EURIDES, D. Preparo de rufiões bovinos por fixação da flexura sigmóide do pênis. Santa Maria, 1981. 37p. Dissertação (Mestrado em Cirurgia) - Curso de Pósgraduação em Medicina Veterinária, Universidade Federal de Santa Maria, 1981

EURIDES, D., CONTESINI, E.A., VIANA, S. M. Preparação de rufiões bovinos por remoção do ligamento apical do pênis. Ciência Rural, Santa Maria, v.22, n.2, p.185-189, 1992.

JENNINGS, JR.P.B. The practice of large animal surgery. Philadelphia : Saunders, 1984. 1233p.

JILLELLA, D., BAKER, A.A., EATON, R.J. Preparation of teaser bulls by dorsal scrotal penile deflection. Aust Vet $\mathbf{J}$, v.54, p.358-359, 1978

JOCHELE, W., GIMENEZ, T., ESPARZA, H., et al. Preparation of teasers bulls ramps na boards by penis and prepuce desviation. Vet Med, v.68, n.4, p.395-400, 1973

LEITE RIBEIRO, A.C.C., ALVES, G.E.S., ABREU , J.J., et al. Uso da acepromazina $1 \%$ como meio auxiliar na exposição do pênis bovino. Rev Bras Reprod Anim , Belo Horizonte, v.8, n.1, p.9-11, 1984.

LOPEZ, J.E., CRUDELI, G.A., TORRES, J.G., et al . Epididymectomy in teaser buffalo bulls. Bubalus-Bubalis, v.5, n.2, p.18-21, 1999

MARQUES, J.A., MARQUES, L.C., CANOLA, J.C. Acropostite - Fimose em touros. Uma técnica cirúrgica de tratamento. Clin Vet, v.2, n.1, p.2-3, 1988.

MOLINARI, C. E., HRDALO, J. C., GIL, O.H. Fijacion de la flexura distal de la $\mathrm{S}$ sigmoidea para la preparacion de toros marcadores de celo (tecnica de Monroy). Nuestra experiencia. Veterinaria-Argentina, v. 15, n.142, p.109-113, 1998

NILSFORS, L., GARMER, L., ADOLFSON, A. Sedative and analgesic effects of medetomidine in dogs - an open clinical study. Acta Vet Scand, v.85, p.155-159, 1988.

OEHME, F.W. Resection of the bovine epididymis. A procedure for preparing teaser bulls and seguring increased weight gain in male feeder cattle. Vet Med, v.63, p.603-606, 1968.

OEHME, F.W. Textbook of large animal surgery. 2.ed. Baltimore : Williams \& Wilkins, 1988. 714p.

ROMMEL, W. Desviacion quirurgica del pene en bovinos confines de detectar el celo en las hembras. In ALEXANDER, A. Tecnica quirurgica en animales. 2.ed., México : Interamericana, 1961. p.231-238.

SAMPAIO, I.B.M. Estatística aplicada à experimentação animal. Belo Horizonte : Fundação de Ensino e Pesquisa em Medicina Veterinária e Zootecnia, 1998. 221p.

SCHULTZ, L.G., BRYANT, L.K., ROSS, G.S., et al. Teaserbull preparation using a penile deviation technique. Comp Cont Educ Pract Vet, v.21, supplement 3, S86-S89, 1999.

SILVA, L.A.F. Preparo de rufião eqüino através da circuncisão com encurtamento do pênis. Avaliação do comportamento sexual. Belo Horizonte, 1992. 133 p. Tese (Doutorado em Ciência Animal) - Escola de Veterinária da UFMG, 1992

SILVA, L.A.F., CHAVES, S.M., FIORAVANTI, M.C.S., et al Utilização da acepromazina associada a xilazina na preparação cirúrgica de rufiões bovinos. In: CONGRESSO BRASILEIRO DE CIRURGIA E ANESTESIOLOGIA VETERINÁRIA, 4, 2000, Goiânia, GO. Ciência Animal Brasileira... Goiânia : UFG, 2000. v.1. p.214.

SMITH, L.C. Surgical procedures to make a bull a heat detector. A I Digest, v.9, p.1, 1963.

SMITH, A.P. Epididymal transection for sterilising bulls. J Am Vet Med Assoc, v.150, n.6, p.633, 1967.

STRAUB, O.C., KENDRICK, J.W. Preparation of teaser bulls by penectomy. J Am Vet Med Assoc, v.147, n.4, p.373-376, 1965

TEGEGNE, A., GELETO, A., KASSA, T. Short luteal phases and ovulations without oestrus in primiparous Borana (Bos indicus) cows in the central highlands of Ethiopia. Animal Reproduction Science, v.31, n.1-2, p.21-31, 1993. 